\title{
A genome-scale mining strategy for recovering novel rapidly-evolving nuclear single-copy genes for addressing shallow-scale phylogenetics in Hydrangea
}

Carolina Granados Mendoza ${ }^{1,2^{*}}$, Julia Naumann ${ }^{3}$, Marie-Stéphanie Samain ${ }^{1,4}$, Paul Goetghebeur ${ }^{1}$, Yannick De Smet ${ }^{1}$ and Stefan Wanke ${ }^{3^{*}}$

\begin{abstract}
Background: Identifying orthologous molecular markers that potentially resolve relationships at and below species level has been a major challenge in molecular phylogenetics over the past decade. Non-coding regions of nuclear low- or single-copy markers are a vast and promising source of data providing information for shallow-scale phylogenetics. Taking advantage of public transcriptome data from the One Thousand Plant Project (1KP), we developed a genome-scale mining strategy for recovering potentially orthologous single-copy markers to address low-scale phylogenetics. Our marker design targeted the amplification of intron-rich nuclear single-copy regions from genomic DNA. As a case study we used Hydrangea section Cornidia, one of the most recently diverged lineages within Hydrangeaceae (Cornales), for comparing the performance of three of these nuclear markers to other "fast" evolving plastid markers.

Results: Our data mining and filtering process retrieved 73 putative nuclear single-copy genes which are potentially useful for resolving phylogenetic relationships at a range of divergence depths within Cornales. The three assessed nuclear markers showed considerably more phylogenetic signal for shallow evolutionary depths than conventional plastid markers. Phylogenetic signal in plastid markers increased less markedly towards deeper evolutionary divergences. Potential phylogenetic noise introduced by nuclear markers was lower than their respective phylogenetic signal across all evolutionary depths. In contrast, plastid markers showed higher probabilities for introducing phylogenetic noise than signal at the deepest evolutionary divergences within the tribe Hydrangeeae (Hydrangeaceae).

Conclusions: While nuclear single-copy markers are highly informative for shallow evolutionary depths without introducing phylogenetic noise, plastid markers might be more appropriate for resolving deeper-level divergences such as the backbone relationships of the Hydrangeaceae family and deeper, at which non-coding parts of nuclear markers could potentially introduce noise due to elevated rates of evolution. The herein developed and demonstrated transcriptome based mining strategy has a great potential for the design of novel and highly informative nuclear markers for a range of plant groups and evolutionary scales.
\end{abstract}

Keywords: Data mining, Fine-scale phylogenetics, Hydrangea sect. Cornidia, Phylogenetic signal, Phylogenetic noise

\footnotetext{
*Correspondence: carolina.granados@st.ib.unam.mx; stefan.wanke@tu-dresden.

de

'Department of Biology, Research Group Spermatophytes, Ghent University,

K.L. Ledeganckstraat 35, 9000 Ghent, Belgium

${ }^{3}$ Institut für Botanik, Technische Universität Dresden, Zellescher Weg 20b,

01062 Dresden, Germany

Full list of author information is available at the end of the article
}

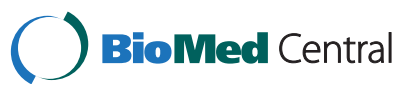

(c) 2015 Granados Mendoza et al. This is an Open Access article distributed under the terms of the Creative Commons Attribution License (http://creativecommons.org/licenses/by/4.0), which permits unrestricted use, distribution, and reproduction in any medium, provided the original work is properly credited. The Creative Commons Public Domain Dedication waiver (http://creativecommons.org/publicdomain/zero/1.0/) applies to the data made available in this article, unless otherwise stated. 


\section{Background}

Identification of informative markers for specific phylogenetic questions finds one of its major challenges at shallow-scale phylogenetics, mostly along the gradient between species and population level. In particular, short internodes in phylogenetic trees, where lineages split in quick succession, are difficult to resolve [1]. Plastid and nuclear ribosomal DNA loci (nrDNA) have predominantly been used in phylogenetic studies of flowering plants [2-7]. However, their applicability for resolving shallow divergences is limited by several characteristics. Plastid markers show reduced sequence divergence at a low phylogenetic level in most plant lineages due to the slow and nearly constant rate of plastome evolution relative to the nuclear genome $[3,8]$. When used as the sole source of information, plastid markers provide only one side of the information about processes such as hybridization and introgression $[9,10]$, since they are inherited maternally in most angiosperms [3]. Nuclear ribosomal DNA markers occur in high numbers of copies, causing problems for assessing orthology $[4,11]$. Furthermore, due to incomplete homogenization of nrDNA gene families (i.e. partial concerted evolution; [12, 13]), biparental inheritance might be traced inconsistently in hybrids $[3,10,14,15]$.

Nuclear single or low-copy genes, and particularly their introns, have the potential to overcome these disadvantages. Compared to plastid markers, they are generally more variable, potentially providing a higher number of parsimony informative characters per sequenced bases $[9,14]$. The biparental inheritance of nuclear markers facilitates the identification of possible hybridization and polyploidization events, and when used in concert with plastid markers the directionally of hybridization can be inferred [9, 10, 14-19]. When present in low- or single-copy, nuclear markers are a highly valuable source of putative orthologous loci, that are increasingly being accessed in plant phylogenomics [7, 9, 10, 15, 19-34]. For a long time in plant phylogenetics, data and technology were unavailable or at least limited to explore and broadly screen the nuclear genome. One of the first genome scale comparative approaches identified a set of 959 nuclear single-copy genes (NSCG) shared by Arabidopsis thaliana, Populus trichocarpa, Vitis vinifera and Oryza sativa (APVO SSC genes; [14]). The utility of these loci has been demonstrated by reconstructing angiosperm phylogenetic relationships from deep to shallower evolutionary depths $[9,14,24]$.

Compared to the functionally constrained exons, the introns of the APVO SSC genes provide additional variability shown to be useful at and below species level [9]. Even though the benefits of employing potential nuclear single-copy genes (NSCG) for plant phylogenetics were highlighted years ago [2, 3, 35], only now are they becoming more popular and widely used with the expansion of nuclear genome resources. Next Generation Sequencing Technologies (NGS) generate an increasing amount of genomic resources [31,36]. This coupled with the recent proliferation of novel genome-partitioning strategies have considerably facilitated the application of NSCG to plant phylogenetic studies [37]. Two highly promising genome partitioning strategies currently applied to plant phylogenetics are anchored hybrid enrichment, currently under test and development, (Alan Lemmon, Florida State University, USA, personal communication) and transcriptome sequencing. Among these two strategies, mining publicly available transcriptome resources for developing novel NSCG markers for non-model plant species is being increasingly applied [29, 38-41]. Recent approaches for the discovery of novel NSCG markers from public or newly produced transcriptome data range from the application of elaborate bioinformatics pipelines $[29,39,40]$ to the development of automated tools (e.g. MarkerMiner; [42]).

International projects, such as the "1000 plants" (1KP, http://www.onekp.com) use more cost efficient strategies such as transcriptome sequencing, compared to whole genome sequencing, and continuously make nuclear genome-scale information accessible for an increasing number of green plants. The present study benefits from these transcriptome resources for identifying novel rapidlyevolving nuclear loci for shallow-scale phylogenetics in $H$. sect. Cornidia. This section of mostly Neotropical species is one of the most recently diverged lineages within the tribe Hydrangeeae (Hydrangeaceae, Cornales; [43-45]) and is composed of 13 currently accepted species and a yet unknown number of new species of evergreen, rootclimbing hortensias [46]. Although resolution and support for the internal relationships of Hydrangea have progressively been improved by the addition of highly variable coding and non-coding plastid regions $[44,45]$ and nuclear ribosomal ITS sequences [43], a number of phylogenetic splits at the species level and below remain unresolved. Previously used plastid markers have shown extremely low variability among studied $H$. sect. Cornidia representatives. This has been attributed to a potential rapid or recent diversification event [44] and could additionally be due to a very slow rate of sexual reproduction. Nuclear single or low-copy sequence data have been generated for only two Hydrangeaceae species in the past, namely Philadelphus incanus (SMC1, SMC2, MSH1, MLH1 and MCM5 genes, [13]) and H. paniculata (Xdh gene, [47]). However, none of these nuclear loci have been employed for a wider taxonomical sampling in the family. All these lines of evidence make $H$. sect. Cornidia an excellent study case for testing the performance of potential NSCG retrieved by a genome scale mining strategy for resolving fine scale phylogenetic relationships.

Methods such as those developed by Townsend [48] and Townsend et al. [49] provide an efficient approach for 
estimating and comparing the utility of different molecular markers for specific phylogenetic questions. Townsend's phylogenetic informativeness (PI) method provides a quantitative measure of the phylogenetic signal in markers by quantifying the probability that a character changes at a certain position of a tree, and does not undergo further change [48]. For this, the evolutionary changes across sites are compared against an ideal change rate based on a reference ultrametric tree, where branches are proportional to evolutionary units. The PI method has been implemented in the freely-accessible online program PhyDesign [50]. This program requires a priori sequence data for the candidate loci and only one reference tree that can, for instance, be calculated from a subset of the taxa of interest. Although the PI method does not account for potential phylogenetic noise, Townsend and collaborators [49] recently proposed an analytical solution for estimating the probabilities of correct, incorrect and polytomous resolutions given rates of evolution and a defined state space.

Taking advantage of these recently published analytical tools, as well as of public genome resources, our aims are to: 1) develop a genome-scale mining strategy for recovering novel, intron-rich, nuclear single-copy loci potentially useful for shallow-level phylogenetics in Hydrangea, 2) design a set of primers for successfully amplifying and sequencing these regions from genomic DNA in Hydrangea, 3) test the success of some of these markers in resolving shallow divergences in $H$. sect. Cornidia, 4) estimate the phylogenetic informativeness of the selected nuclear markers and compare them with other previously used "fast" evolving plastid markers: the $r p l 32-n d h F$ intergenic spacer (IGS), $\operatorname{trn} V-n d h C$ IGS, trnL-rpl32 IGS and $n d h A$ intron, and 5) compare the performance of nuclear and plastid markers for resolving shallow and deeper-level divergences in Hydrangea.

\section{Results}

\section{Mined potential NSCG}

Our data mining process retrieved 4949 Cornales scaffolds that could be aligned to $546 \mathrm{~A}$. thaliana single-copy gene orthologs. This pool of genes was used to select the most promising and the easiest to handle markers for $H$. sect. Cornidia. We were aiming for gene alignments where a major part of the gene was covered by at least four of the $1 \mathrm{KP}$ Cornales species. This is crucial for estimating phylogenetic utility of the candidate markers, as well as for successful primer design. Among the 546 initial alignments, 444 showed low coverage of Cornales representatives and additional 16 showed low coverage of the two Hydrangeeae taxa (Fig. 1). Therefore these alignments were not considered any further. Multiple scaffold sequences were detected for $H$. quercifolia in 13 of the remaining alignments and were therefore excluded from further steps. A total of 73 potential NSCG were retained after filtering (Fig. 1). From these alignments, 14 contained the six Cornales representatives, whereas 53 lacked either Curtisia dentata or Deutzia scabra and six did not have either Philadelphus inodorus and Curtisia dentata, or Curtisia dentata and Deutzia scabra. When considering transcriptome sequences from all Cornales taxa, alignments showed 26.7-62.3\% variable sites (Fig. 2). However, the range of variable sites was lowered to 19.8-57.5 \% when only the four Hydrangeaceae representatives were considered and to $2.8-26.9 \%$ between the two representatives of tribe Hydrangeeae (Fig. 2).

\section{Amplification and sequencing of selected NSCG}

From the 27 tested primer pairs, 11 yielded amplicons with single bands for the reduced taxon sampling. The remaining 16 primer combinations either produced multiple bands or did not amplify in either $H$. sect. Cornidia or the outgroup taxa. Primer pairs producing multiple PCR products corresponded to the AT2G17975, AT5G48470, AT3G54170 and AT5G12040 A. thaliana orthologs. Among the regions that amplified a single band, only three produced clear sequences, and were therefore selected for further tests using the extended taxon sampling. The amplified regions correspond to portions of the A. thaliana gene orthologs AT1G10840, AT1G63900 and AT5G57410 (Fig. 3). According to TAIR (http://www.arabidopsis.org/), the AT1G10840 ortholog corresponds to the gene TIF3H1 (Translation initiation factor 3 subunit $\mathrm{H} 1$ ) encoding the eukaryotic initiation factor $3 \mathrm{H} 1$ subunit and is part of the eukaryotic translation initiation factor 3 (eIF-3) complex. The AT1G63900 gene, known as the DAL1 (DIAP1-like protein 1) or SP1 (Suppressor of PPI1 locus 1 ) gene (hereafter referred to as $D A L 1$ gene) encodes a RING-type ubiquitin E3 ligase present in the chloroplast outer membrane. The AT5G57410 ortholog is known to encode a microtubule-associated protein. Gene Ontology annotations [51] for these genes are provided in Additional file 1 . A region of ca. $430 \mathrm{bp}$ located in the $5^{\prime}$ portion of the amplified DAL1 gene region was excluded from the final alignments because long mononucleotide repeats yielded low quality sequences. Compared to A. thaliana, coding regions in $H$. sect. Cornidia species show high sequence similarity, while non-coding regions were overall considerably greater in length (Fig. 3).

\section{Phylogenetic relationships of Hydrangea sect. Cornidia}

Our combined nuclear and plastid data set comprised a total of 7994 characters. Due to uncertain homology assessment, 357 characters were removed from subsequent analyses. Positions of excluded regions in the initial alignment, as well as important characteristics from each nuclear and plastid data subsets are shown in Additional file 2. With the exception of two internal nodes indicated below, our total combined nuclear and plastid ML analysis retrieved strongly supported clades with $\mathrm{BS} \geq 85$ 


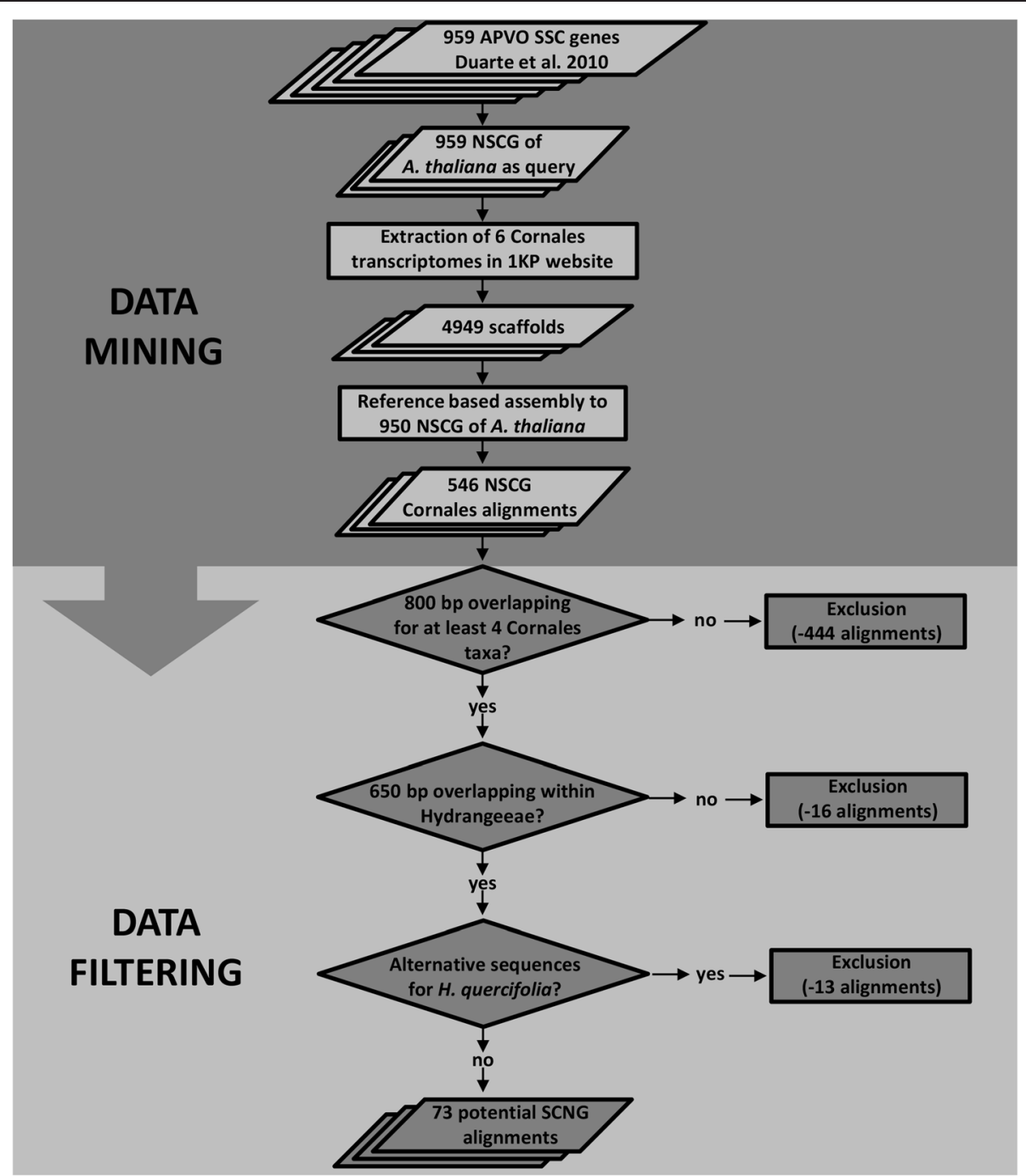

Fig. 1 Nuclear single-copy gene mining strategy and data filtering. Data mining and filtering strategy employed for identifying potential NSCG for resolving shallow evolutionary divergences in $\mathrm{H}$. sect. Cornidia

(Fig. 4). No statistically supported incongruences were detected between the combined plastid and combined nuclear analyses. However, the nodal support based on the combined nuclear dataset was remarkably higher than that retrieved with the combined plastid matrix (Additional file 3).

Hydrangea sect. Cornidia was recovered as monophyletic with $H$. anomala of section Calyptranthe as its sister species. Within $H$. sect. Cornidia three main clades were recovered. The first of them $(\mathrm{BS}=56)$ consisting of $(H$. integrifolia $(H$. seemannii $+H$. serratifolia) $)$ is sister to a lineage containing the other two main clades. In the second main clade an undescribed species from Costa Rica (H. sp. 3 ) is sister to a clade that further divides into two groups. The first of these groups includes a grade of $H$. steyermarkii, $H$. nebulicola and two undescribed Mexican species ( $H$. sp. 1 and $H$. sp. 2), while the second is formed by $H$. oerstedii sister to $H$. aff. peruviana (Fig. 4). The third main clade consists of a grade of $H$. preslii, $H$. tarapotensis, $H$. jelskii and $H$. sprucei plus an undescribed Ecuadorian species (H. sp. 4). The phylogenetic position of $H$. tarapotensis, however, received low statistical support $(\mathrm{BS}=65)$.

\section{Phylogenetic informativeness of NSCG and plastid markers}

Per site PI of NSCGs was up to threefold higher than that of the plastid markers rpl32-ndhF IGS, $\operatorname{trn} V-n d h C$ IGS, trnL-rpl32 IGS and $n d h A$ intron (Fig. 4). The three NSCGs showed highly similar per site PI profiles with a steep increase during the shallowest divergences within $H$. sect. Cornidia and followed by a more steady increase reaching their $\mathrm{PI}_{\max }$ at the times 0.76 (AT5G57410), 0.77 (DAL1) and 0.81 (TIF3H1; Fig. 4). Lower, rather flat profile curves were recovered for the four plastid regions 


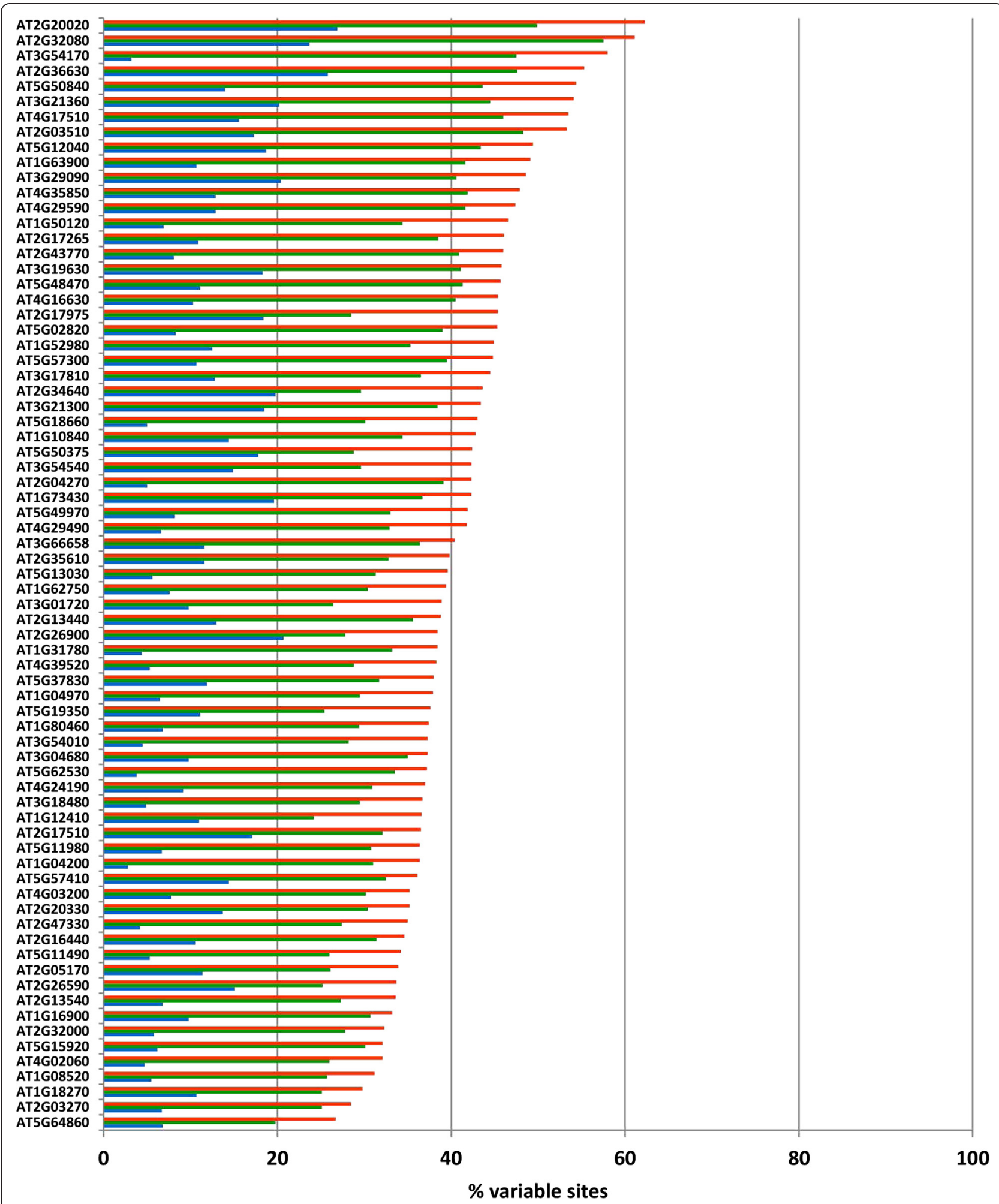

Fig. 2 Retained potential NSCG after the entire data mining and filtering process. Genes have been ordered by their percent of variability among Cornales representatives. Orange bars denote the percent of variable sites among the six Cornales taxa, whereas green and blue bars represent the percent of variable sites among the four Hydrangeaceae representatives and the two tribe Hydrangeeae taxa, respectively 

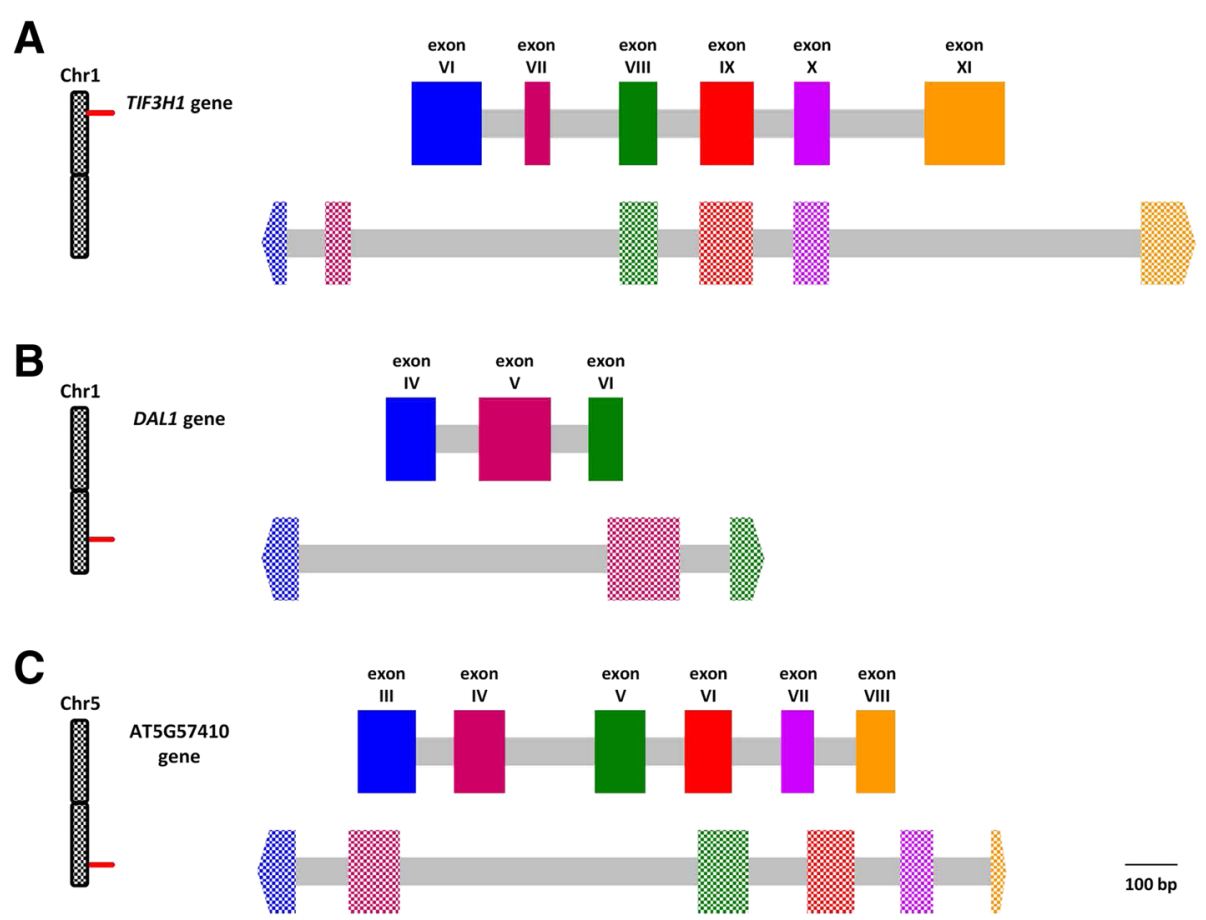

$\overline{100 \mathrm{bp}}$

Fig. 3 Nuclear single-copy gene models. Models for the amplified portions of the TIF3H1 (a), DAL1 (b), and AT5G57410 (c) genes used for resolving shallow evolutionary divergences in $\mathrm{H}$. sect. Cornidia. Lengths of the respective regions are drawn as found in H. seemannii (Granados M. et al. 479, Pueblo Nuevo, Durango, Mexico). Solid colored large boxes identify exon regions of $A$. thaliana, whereas dotted colored large boxes denote exon regions of $H$. seemannii. Homology of exon regions between the two species is indicated by common colors. Non-coding regions are indicated by grey boxes. Relative position of each gene within the respective A. thaliana chromosome is shown as indicated in TAIR (http://www.arabidopsis.org/)

(Fig. 4). The curves of the plastid markers show a moderate initial increase and a flatter shape reaching their $\mathrm{PI}_{\max }$ at the times 0.79 (rpl32-ndhF IGS), 0.82 (ndhC$\operatorname{trn} V$ IGS) and 0.88 (rpl32-trnL IGS; Fig. 4). Only the $n d h A$ intron reached its $\mathrm{PI}_{\max }$ considerably earlier (at time 0.53) than any other plastid or nuclear marker, leading to the lowest per site PI values (Fig. 4).

\section{Performance of nuclear and plastid markers in resolving shallow and deeper-level divergences}

The phylogenetic signal and noise analysis was applied to assess the probability of the nuclear and plastid data sets resolving shallow and deeper-level divergences. As compared to our reference tree resulting from the combined analysis of all nuclear and plastid markers uploaded in PhyDesign, nuclear single copy genes retrieved considerably higher probabilities of correct over incorrect resolutions for shallow nodes; these probabilities become more similar towards deeper nodes (Fig. 4). In contrast, plastid markers recovered similar probabilities of correct and incorrect resolutions from the shallowest nodes and lower probabilities of correct resolution relative to incorrect resolution at the two deepest nodes (Fig. 4). The probability of polytomous resolution was considerably higher for the plastid relative to the nuclear data set across all nodes.

\section{Discussion}

\section{Genome-scale mining strategy}

Large amounts of publicly available genome-scale data from a broad variety of green plants offer new and promising prospects in plant systematics. The constantly increasing public resources of nuclear sequence data provide a vast pool for nuclear marker development. It is now possible to identify orthologous nuclear markers for nearly any plant group of interest and for a wide range of phylogenetic levels. The transcriptome mining approach described here for Hydrangeaceae is highly efficient and powerful for identifying novel nuclear single-copy genes informative at shallow evolutionary depths. A pool consisting of hundreds of nuclear candidate genes can quickly be reduced to a set of best and most efficient markers for a specific phylogenetic question following a few simple criteria. Specifically, we targeted orthologous genes with intron-rich regions that can be amplified broadly and sequenced from genomic DNA. A high number of initial candidate genes allowed the efficient discarding of less promising phylogenetic markers and still left a good number of potentially orthologous, highly informative nuclear loci.

Among the excluded nuclear genes, we identified a handful with different sequence variants for $H$. quercifolia, suggesting multiples copies for these nuclear genes in this species and potentially in other Hydrangea species. Several 


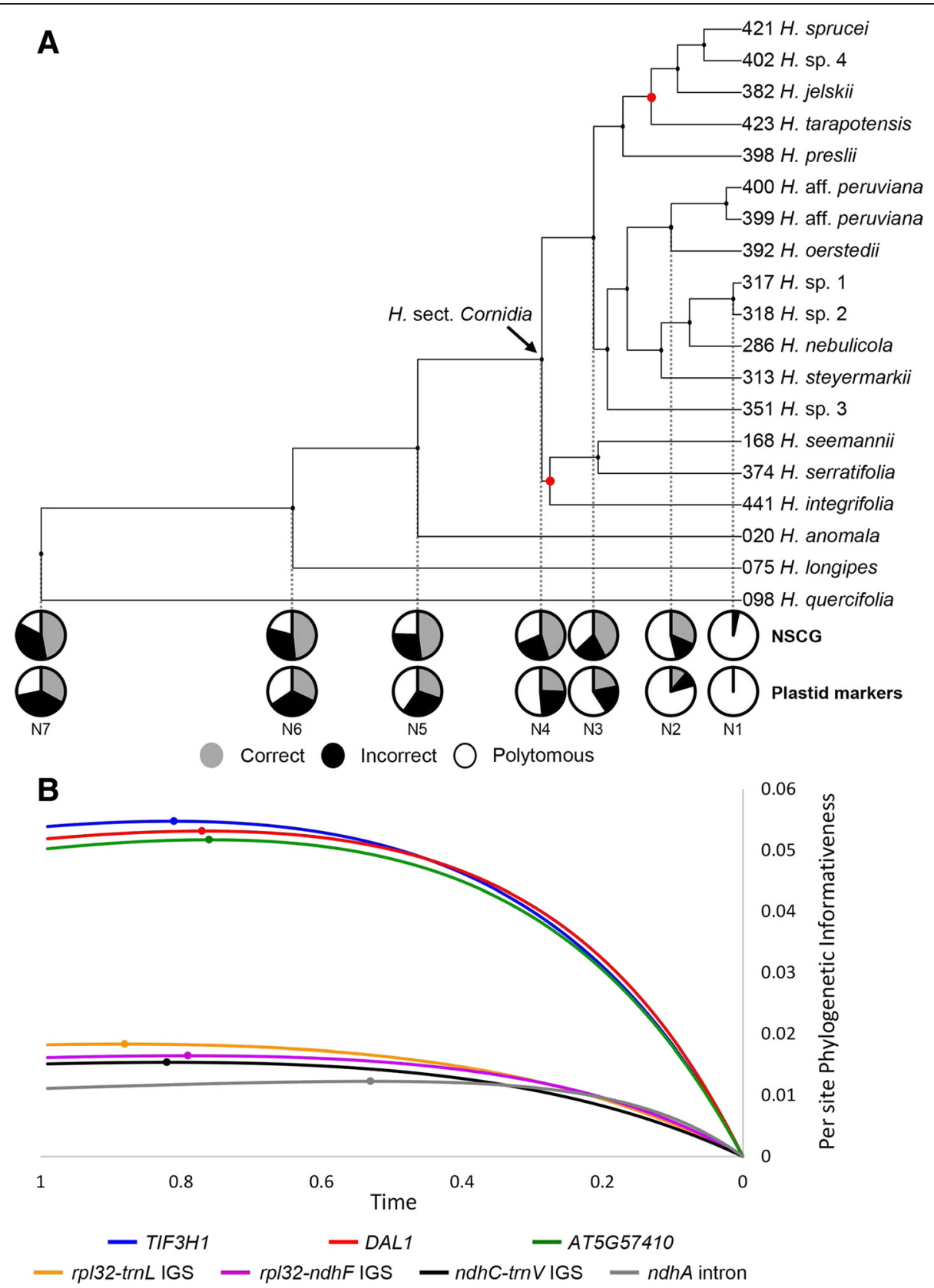

Fig. 4 Phylogenetic informativeness, signal and noise of nuclear and plastid markers. Pie charts show the relative probabilities of correct (grey), incorrect (black) and polytomous (white) resolution of nuclear and plastid datasets across a range of individual nodes (N1-N7) within the phylogeny of H. sect. Cornidia and its close relatives. The probability of nuclear markers recovering incorrect resolutions is lower than their respective probability of recovering correct resolutions across all nodes. In contrast, plastid markers show higher probabilities of recovering incorrect than correct resolutions at the deepest nodes (N5 to N7; a). Per site PI across time of the individual nuclear and plastid regions (b). Red circles indicate BS $<85$ for internal nodes

factors can explain the observed potential gene duplications. First, all flowering plants are ancestral polyploids [52] and polyploidization events are common among angiosperms lineages $[34,53,54]$. In addition to the gamma polyploidization, predating the diversification of the core Eudicots, three additional polyploidization events are known to have occurred in the Asterid clade according to genome wide comparisons $[55,56]$. Second, alterations to the basic chromosome number in Hydrangea $(2 \mathrm{n}=36)$ are known as a result of descending dysploidy in the $H$. aspera complex $(2 \mathrm{n}=30$ and $[34,57,58])$. Since descending dysploidy is frequently generated by chromosome rearrangements such as centromere losses in monocentric chromosomes resulting in chromosome number reduction [59], little effect could be expected on the number of copies of a gene (gene dosage) according to Duarte et al. 
[14]. However, descending dysploidy is known to frequently follow polyploidy events [59], which in this case could have preceded the diversification of the genus. A specific polyploidy event predating the diversification of Hydrangea could explain the multicopy nature of some APVO genes [14] in $H$. quercifolia $(2 \mathrm{n}=2 \mathrm{x}=36$; [57]). Previously reported cases of triploids ( $H$. macrophylla), tetraploids $(H$. liukiuensis), hexaploids (D. febrifuga), octoploids and decaploids ( $H$. yayeyamensis; $[60,61]$ ) sustain the notion of polyploidy being a frequent evolutionary event in the hortensias and their relatives.

Due to the large number of initial candidate genes, discarding those with multiple variants, did not limit our power for answering our phylogenetic question. However, researchers working with a limited amount of candidate markers may opt for determining the origin of such sequence variants (e.g. occurrence of alternative splicing of pre-mRNA molecules). If the observed sequence variants are not discovered at the genomic DNA level, they might be splice variants, and the specific marker could be retained. Otherwise, multiple genomic variants indicate gene duplication and the marker should be excluded from further phylogenetic analyses. For this reason, a large repertory of initial candidate genes is highly beneficial.

Another important advantage of our mining strategy is its broad applicability. The 73 potential NSCG could potentially be useful for other Cornales families since the genome-scale mining strategy implemented here included representatives from two other Cornales lineages. Additionally, our taxon sampling could easily be expanded by adding further lineages from outside Cornales. Our markers are likely useful at a range of taxonomic levels due to the observed exon variability. Moreover, our data mining and filtering strategy can be applied to any other plant group with enough genome resources. Hence, because of its versatility, this method has the potential to be used by a wide range of plant evolutionary biologists.

\section{Establishment of selected nuclear single-copy markers}

The next relevant step in marker development, after potential candidates have been selected, is to screen for successful primer combinations and to test their applicability. A number of tested primer pairs did not yield PCR products. These primer combinations were designed based on exon sequences of six Cornales representatives including $H$. quercifolia. Since the latter species was included in our test taxon sampling, it is unlikely that amplification failure is due to the primer design. A more plausible explanation could be differences between $A$. thaliana and $H$. sect. Cornidia intron sizes. While exon size is highly conserved, intron size is considerably greater in $H$. sect. Cornidia relative to $A$. thaliana. These observations are in agreement with the 1C DNA content of A. thaliana and Hydrangea diploids which range from $0.05-0.23 \mathrm{pg}$ and $0.98-3.5 \mathrm{pg}$, respectively (Plant DNA C-values Database, release 6.0, December 2012, http://data.kew.org/cvalues/). The differences in intron size and $1 \mathrm{C}$ DNA content suggest that a number of primer pairs fail to amplify because they likely spanned long introns that could not be amplified using regular PCR conditions.

Primer pairs belonging to four different genes were found to amplify multiple, two to four, PCR products of different sizes in $H$. quercifolia and one or more species from our test taxon sampling. These multiple PCR products could be the result of the amplification of more than one gene region due to low primer specificity, as well as the amplification of a multicopy gene region. In neither of these cases the primer pairs could be considered as good candidates, hence we excluded them from further tests.

Among retained potential single-copy candidate markers, three regions amplified a single band and produced clear sequence reads. The majority of the other regions showed multiple peaks for a nucleotide in the chromatograms, most likely as a result of multiple copies of similar size or differences among alleles, or long mononucleotide repeat units followed by a decrease in sequence quality. Sequence regions divided by highly repetitive elements, such as long mononucleotide repeats, are hard to bridge during sequencing and, if "successfully" sequenced, the number of repetitive elements is always uncertain, even when using certain next generation sequencing approaches (e.g. 454 pyrosequencing; [62]). Therefore, regions containing highly repetitive motifs are preferably removed from marker selection processes [63].

\section{Phylogenetic utility of nuclear single copy genes for the case of $\mathrm{H}$. sect. Cornidia}

Our total combined plastid and nuclear analysis obtained a fully resolved and highly supported phylogenetic hypothesis, with the exception of the affinities of $H$. integrifolia and $H$. tarapotensis. Hydrangea integrifolia is connected to the rest of the $H$. sect. Cornidia species by a short and deep internode. Such internodes are a common pitfall in plant phylogenetics [64] and generally require the inclusion of multiple markers that are highly informative for that specific divergence time scale $[1,24,45]$. A long branch leads to $H$. integrifolia, as well as each of the species $H$. seemannii and $H$. serratifolia -here recovered as closely related. These long branches are potentially the result of accelerated evolutionary rates due to relaxed evolutionary constraint of the non-coding regions (i.e. introns), which could promote sequence saturation and result in long-branch attraction among these three species (see [65]). Future studies should, however, include a more detailed data partitioning scheme that separates non-coding from coding regions, as well as 1st, 2nd and 3rd codon positions among coding regions, since it has been shown that moderate to high rates of substitution in coding regions do 
not always negatively affect phylogenetic reconstruction [66]. Inclusion of more taxa has been shown to break long branches [67]. Addition of other $H$. sect. Cornidia species might help reduce the potential long-branch attraction effect of $H$. integrifolia and help elucidate its affinities. Specifically, in the case of $H$. tarapotensis, internode and branch length does not seem to explain its unsupported affinities and none of the plastid and nuclear data recovered strongly supported relationships for this species. Inclusion of more informative data near the specific time scale of divergence of $H$. tarapotensis may help clarify its phylogenetic relationships.

Plastid and nuclear data did not show strongly supported incongruence justifying the viability of a total combined analysis. Both plastid and nuclear data retrieved strong support for the deepest divergences; however, nodal support for shallower divergences retrieved from the combined nuclear analysis surpassed by far that from the combined plastid regions. The latter suggests that the nodal support obtained for the low-level divergences was provided by phylogenetic signal mostly contained in the nuclear data.

\section{Phylogenetic informativeness of NSCG and plastid markers}

Per site PI profiles of the nuclear markers were in general much higher than those of plastid markers. To our knowledge, no previous study has compared PI profiles of nuclear single-copy and plastid data. From our comparison we can observe considerably higher phylogenetic utility of nuclear single-copy genes than plastid markers at most of the evolutionary depths of our phylogenetic framework. All nuclear and plastid markers peaked deeper than the time of divergence of $H$. sect. Cornidia, suggesting that little or low phylogenetic noise can be expected along the evolutionary time scale of $H$. sect. Cornidia. At deeper taxonomical levels, potential phylogenetic noise, as indirectly inferred from PI profiles, can be expected from the $n d h A$ intron around the divergence time of $H$. anomala, and from the rest of the markers close to the divergence time of $H$. longipes. Special attention should be paid to the $n d h A$ intron since despite its low variability, this plastid IGS peaked even earlier than the more variable nuclear markers. A combined plastid and nuclear analysis excluding the $n d h A$ intron (Additional file 3) retrieved the same phylogenetic relationships as our total combined plastid and nuclear analysis; however, the bootstrap support for the sister relationship of $H$. nebulicola and the two undescribed Mexican species increased from 86 to 93. The latter suggests that the $n d h A$ intron not only provides low phylogenetic signal at shallow evolutionary depths, but also potential phylogenetic noise for deeper divergences in Hydrangea.

\section{Ability of NSCG and plastid markers for resolving shallow and deeper-level divergences}

A recent angiosperm-wide study by Han et al. [53] found that the addition of single-copy gene introns resulted in increased branch support, although it also uncovered notable incongruences. Based on this finding, Han et al. [53] recommended the use of single-copy intron sequences for lower taxonomic levels. Intron variability within our nuclear data set also seems to present the appropriate evolutionary pace for resolving shallow divergences in $H$. sect. Cornidia without introducing phylogenetic noise. Plastid markers might be more appropriate for deeper taxonomic levels, at which non-coding regions of nuclear markers could potentially introduce phylogenetic noise. Future studies implementing highly efficient techniques for the collection of high-throughput sequencing data (e.g. [63]) should consider analyzing these larger data sets under a scheme that accounts for possible gene-tree incongruences and phylogenetic noise potentially introduced by numerous fast-evolving sites [68].

\section{Conclusion}

The genome-scale mining strategy developed and demonstrated here successfully recovered a number of novel, rapidly-evolving nuclear single-copy genes useful at different taxonomic depths not only within Hydrangea, but with potential for use in other Cornales taxa and beyond. Other transcriptome mining strategies such as that developed by Rothfels et al. [29], Tonnabel et al. [39] and Pillon et al. [40] confirm the great potential of these approaches for developing highly informative markers for phylogenetic reconstructions of different plant lineages and at a range of taxonomic depths. The automation of such transcriptome mining procedures by Chamala et al. [42] certainly represents a valuable contribution to the plant evolutionary research community, allowing the application of these strategies for researchers with little knowledge on bioinformatics. The $1 \mathrm{KP}$ plant transcriptomes are an invaluable source of information for developing orthologous nuclear markers that can complement established conventional markers and help resolve currently unknown evolutionary relationships among green plants. The application of other highly efficient genome partitioning strategies such as the anchored hybrid enrichment, which can potentially allow for the recovery of hundreds of orthologous single copy nuclear loci, is another exciting and promising possibility for upcoming plant phylogenetic studies.

\section{Methods}

Nuclear single-copy gene mining strategy and data filtering As a starting point we used the 959 APVO SSC genes of Duarte et al. [14]. From these, a fasta file containing the coding DNA sequences of the 959 A. thaliana NSCG orthologs was used as query to blast against six Cornales 
species available on the OneKP Project website (blastn, expectation value of 1e-5, sample IDs: QURC-Dichroa febrifuga, ZETY-Hydrangea quercifolia, BTZX-Philadelphus inodorus, OTAN-Deutzia scabra, UZNH-Curtisia dentata and VTLJ-Caiophora chuquitensis; Fig. 1). To obtain alignments for each gene ortholog, the extracted transcriptome sequences were assigned and aligned using the full length genomic sequences of the 959 A. thaliana NSCG as a reference ("map to reference"-tool in Geneious ${ }^{\circ}$ version 7.1.5, Biomatters; Fig. 1). In order to select appropriate markers for primer design, the resulting 546 gene alignments were first screened for the following criteria (Fig. 1): 1) only alignments containing more than 800 bp continuously overlapping for at least four Cornales taxa were retained, 2) alignments with less than 650 bp overlapping between $H$. quercifolia and D. febrifuga were excluded and 3) in order to avoid potential multiple copy regions, alignments presenting scaffolds with alternative sequences for $H$. quercifolia were excluded from further steps. Hydrangea quercifolia is the closest relative of $H$. sect. Cornidia among the six Cornales species.

\section{Primer design, amplification and sequencing}

The initial alignments contained the mined transcriptome sequences only, thus providing no information about the potential intron length and variability. For this reason, the following ten candidate nuclear loci were randomly selected from the pool for primer design: AT1G10840, AT1G63900, AT2G17975, AT3G54170, AT4G35850, AT5G12040, AT5 G13030, AT5G48470, AT5G57410 and AT5G64860. These genes were subsequently aligned to the A. thaliana full length genomic sequence obtained from TAIR (http:// www.arabidopsis.org/). Alignments were screened for regions with high abundance of non-coding regions. Primers were placed in conserved exons and designed manually.

A total of 27 primer combinations (Additional file 4) were initially tested for amplification and sequencing success on a reduced taxon set representative of the diversity within the Hydrangea I clade [sensu 44], which contains $H$. sect. Cornidia (see Additional file 5). Based on amplification and sequencing results, portions of three nuclear gene orthologs of A. thaliana (AT1G10840, AT1G63900 and AT5G57410) were chosen for testing their phylogenetic performance in a broader taxonomical sampling as described below.

\section{Taxon sampling}

The extended taxon sampling consists of 19 accessions (Additional file 5) including 11 currently accepted $H$. sect. Cornidia species, as well as four undescribed species that we discovered during our field work throughout the Neotropical distribution area of the section. Additionally, $H$. anomala, which has been recovered as the sister species of $H$. sect. Cornidia [43-45], one species from the $H$. aspera complex and $H$. quercifolia were used as the outgroup. We rooted the phylogenetic tree with the latter species since it is positioned in a grade with $H$. arborescens and sister to the clade including the $H$. aspera complex, $H$. anomala and $H$. sect. Cornidia $[43,45]$. Voucher information and GenBank accession numbers are provided in Additional file 5.

\section{Selection of plastid markers}

For comparative purposes the plastid regions $r p l 32-n d h F$ IGS, trn $V-n d h C$ IGS, trnL-rpl32 IGS and $n d h A$ intron were additionally sequenced for all species of our extended taxon sampling. These loci have previously been proposed to be the best candidates for phylogenetic studies within the tribe Hydrangeeae [45]. All plastid regions were amplified using published primers [45].

\section{Molecular methods}

Extraction of genomic DNA was performed from fresh or silica dried leaf material using the DNeasy Plant Mini Kit (Qiagen $\mathrm{GmbH}$ ) or a standard CTAB method [69]. PCR reactions included $8 \mu \mathrm{l}$ dNTP (Carl Roth + Co.KG, $1.25 \mathrm{mM}$ each), $10 \mu \mathrm{l} \mathrm{Taq} \mathrm{buffer}\left(\mathrm{GoTaq}^{\circ}\right.$ Reaction Buffer, $7.5 \mathrm{mM} \mathrm{MgCl} 2), 2 \mu \mathrm{l}$ of each primer $(10 \mathrm{pmol} / \mu \mathrm{l})$, $0.25 \mu \mathrm{l}$ of Taq DNA polymerase (GoTaq ${ }^{\circledR}$ DNA Polymerase, $5 \mathrm{u} / \mu \mathrm{l})$ and $0.5-2 \mu \mathrm{l}$ of DNA template. Water was added accordingly to obtain a total volume of $50 \mu \mathrm{l}$. Amplification was conducted on a T3 Thermocycler (Biometra). Potential nuclear single-copy regions were amplified using a touchup gradient PCR program (Rowther et al., 2012), including an initial denaturation at $94{ }^{\circ} \mathrm{C}$ for $2 \mathrm{~min}$, a loop of 10 cycles repeated 5 times consisting on denaturation at $96{ }^{\circ} \mathrm{C}$ for $45 \mathrm{~s}$, annealing at a temperature gradient (starting $5{ }^{\circ} \mathrm{C}$ below the optimal annealing temperature for each primer pair and increasing $0.5{ }^{\circ} \mathrm{C}$ every cycle) for $30 \mathrm{~s}$ and extension at $72{ }^{\circ} \mathrm{C}$ for $1 \mathrm{~min} / \mathrm{kb}$ of expected length. A final extension at $72{ }^{\circ} \mathrm{C}$ for $7 \mathrm{~min}$ was applied. Plastid markers were amplified following standard protocols adopted from Samain et al. [44]. PCR products were run on a $1.2 \%$ agarose gel and subsequently purified using a gel extraction kit (Macherey \& Nagel). Sequencing was performed by Macrogen Europe sequencing services.

\section{Tree reconstruction}

Maximum Likelihood (ML) was used for phylogenetic reconstructions with the following data partitions: 1 ) each individual NSCG and plastid marker, 2) all NSCG combined, 3) all plastid markers combined and 4) all NSCG and plastid markers combined. Analyses were performed with RAxML v7.2.6 [70] implementing the $\mathrm{GTR}+\Gamma$ nucleotide model as recommended in the manual, using the "rapid bootstrapping and search for the best-scoring ML tree" algorithm [71]. Bootstrap 
replicates were set to 1000 for all analyses. Obtained phylogenetic trees were compiled and drawn using FigTree version 1.4.0 [72].

\section{Estimation of phylogenetic informativeness and nodal support}

We applied the phylogenetic informativeness method [48] and phylogenetic signal and noise analysis [49] in order to evaluate the performance of all the nuclear and plastid regions. All partitions were explored for per site PI [48], as well as phylogenetic signal and noise [49] using the online application PhyDesign [50]. The phylogenetic tree obtained from the total combined nuclear and plastid data matrix was considered as the most reliable phylogenetic hypothesis and used as reference tree in PhyDesign, for this we converted it to "ultrametric" with the program TreeEdit v1.0a10 [73], using the nonparametric rate smoothing method [74]. This tree was subsequently rescaled assigning branch tips to time 0 and root to time 1 . This modified ultrametric tree was then uploaded along with each individual nuclear and plastid data set to the PhyDesign web application. As recommended by the PhyDesign authors, the program HyPhy [75] was used for estimating DNA sequences substitution rates. We applied the best fitting models of substitution for each partition as estimated by the FindModel tool of the HCV Database Project (http:// www.hiv.lanl.gov). For this, initial trees were constructed using both Weighbor [76] and PAUP version 4.0b10 ([77]; Additional file 1). PI profiles for each individual region were contrasted with the reference ultrametric tree. Maximum per site phylogenetic informativeness (PImax) values were recorded for each region in order to determine the time at which each marker is phylogenetically most informative. Additionally, nodal support of the combined nuclear and plastid datasets were estimated by dividing the number of statistically supported clades a with $\mathrm{BS} \geq 85$ by the number of possible bipartitions (i.e. the number of terminals minus three).

\section{Probability of correct, incorrect and polytomous resolution across nodes}

To estimate the ability of nuclear and plastid markers for resolving shallow and deeper-level divergences in $H$. section Cornidia, we performed a phylogenetic signal and noise analysis as implemented in the PhyDesign web application and following the analytical solution of Townsend et al. [49]. For this, the probabilities of correct, incorrect and polytomous resolution given the estimated DNA sequence substitution rates and a state space of four were calculated for the nuclear and plastid data partitions at seven individual nodes: $\mathrm{N} 1(\mathrm{t}=0.001, \mathrm{~T}=0.011), \mathrm{N} 2$ $(\mathrm{t}=0.01, \mathrm{~T}=0.1), \mathrm{N} 3(\mathrm{t}=0.021, \mathrm{~T}=0.210), \mathrm{N} 4(\mathrm{t}=0.028$, $\mathrm{T}=0.284), \mathrm{N} 5(\mathrm{t}=0.046, \mathrm{~T}=0.462), \mathrm{N} 6(\mathrm{t}=0.064, \mathrm{~T}=$
0.640) and $\mathrm{N} 7(\mathrm{t}=0.1, \mathrm{~T}=1)$; where $\mathrm{t}=$ short deep internode length and $\mathrm{T}=$ length of the subtending branch.

\section{Ethics}

The present study does not involve humans, human data or animals.

\section{Data accessibility}

GenBank accession numbers of the sequences used in the present study are provided in the additional file 5 .

\section{Additional files}

\begin{abstract}
Additional file 1: Models of substitution and Gene Ontology annotations. Word file listing the best fitting models of substitution for each data partition applied in the analysis of phylogenetic informativeness, as well as Gene Ontology annotations for selected NSCG used to infer phylogenetic relationships among $H$. sect. Cornidia species.
\end{abstract}

Additional file 2: Regions excluded from phylogenetic analyses and informativeness, signal and noise estimations. Excel file containing the position of excluded regions in the initial alignment and characteristics of each nuclear and plastid data matrix.

Additional file 3: Phylogenetic trees obtained from the analyses of each data partition. PDF file showing the phylogenetic trees obtained from the analysis of each individual nuclear and plastid region, the combined plastid data, the combined nuclear data and the total combined nuclear and plastid data sets.

Additional file 4: Primers used for testing the ease of amplification and sequencing of 10 potential single-copy genes for $\mathrm{H}$. sect. Cornidia. Word file listing all primers that were specifically designed for this study. Non-coding coverage and spanned lengths correspond to that in A. thaliana. Primer combinations denoted in bold correspond to gene regions selected by their ease of amplification and sequencing.

Additional file 5: GenBank accession numbers and voucher information. Excel file detailing the GenBank accession numbers and voucher information associated with the extended and reduced taxon samplings.

\section{Competing interests}

The authors declare that they have no competing interests.

\section{Authors' contributions}

CGM, JN, M-SS and SW conceived and designed the study. CGM and M-SS designed the taxon sampling and CGM, M-SS and YDS collected samples. JN performed the data mining and CGM the data filtering processes of the genome-scale mining strategy. CGM and SW designed the primers. CGM set the laboratory methods and CGM and YDS performed the laboratory work. CGM performed all the analyses and drafted the manuscript. JN, M-SS, PG, YDS and SW proof read the manuscript. All authors read and approved the final manuscript.

\section{Acknowledgments}

Financial support for this study comes from the Special Research Fund of Ghent University (Bijzonder Onderzoeksfonds project 01J03309) and the "Bundesministerium für Bildung und Forschung (BMBF) KMU-innovativ 9: Biotechnologie - BioChance". Seed grants provided to CGM \& SW by the Biology Department of the TU Dresden are gratefully acknowledged. Additional financial support to CGM was granted by the Consejo Mexiquense de Ciencia y Tecnología (Mexico). We acknowledge support by the German Research Foundation and the Open Access Publication Funds of the TU Dresden. The exchange of research group members was aided by a DAAD/CONACYT bilateral agreement between the Instituto de Ecología, A.C. and the TU Dresden. We thank the authorities of Ecuador, Mexico and Peru for permission to collect material (permits no. Ecuador: 001-12-IC-FLO-DNB/MA, Mexico: SGPA/DGGFS/ 712/3801/10 and Peru: 0271-2011-AG-DGFFS-DGEFFS). We are very grateful to 
Hilda Flores, Helga Ochoterena, Esteban M. Martínez, Diana Fernández, Rigoberto R. Rivera, Joaquina Albán, Asunción A. Cano, Rocío Rojas, Mauricio Cisternas, Alexandra Stoll and Alicia Marticorena for their indispensable support during the realization of our fieldwork. We sincerely thank Christoph Neinhuis for providing the research environment at the TU Dresden and supporting our collaborative Hydrangea s. I. research initiative. Thanks to Chantal Dugardin and Rosette Heynderickx who helped with obtaining material and with administrative tasks for this project. Lab work assistance by Juliane Kammerich and Romy Eckart is gratefully acknowledged. Finally, we thank the helpful comments on the manuscript of Isabel Larridon and David Gernandt.

\section{Author details}

'Department of Biology, Research Group Spermatophytes, Ghent University, K.L. Ledeganckstraat 35, 9000 Ghent, Belgium. ²Departamento de Botánica, Instituto de Biología, Universidad Nacional Autónoma de México, Apartado Postal 70-367, 04510 Coyoacán, Distrito Federal, Mexico. Institut für Botanik, Technische Universität Dresden, Zellescher Weg 20b, 01062 Dresden, Germany. ${ }^{4}$ Instituto de Ecología, A.C., Centro Regional del Bajío, Avenida Lázaro Cárdenas 253, 61600 Pátzcuaro, Michoacán, Mexico.

Received: 11 March 2015 Accepted: 9 June 2015 Published online: 04 July 2015

\section{References}

1. Townsend JP, Leuenberger C. Taxon sampling and the optimal rates of evolution for phylogenetic inference. Syst Biol. 2011;60:358-65.

2. Small RL, Ryburn JA, Cronn RC, Seelanan T, Wendel JF. The tortoise and the hare: Choosing between noncoding plastome and nuclear Adh sequences for phylogeny reconstruction in a recently diverged plant group. Am J Bot 1998;85:1301-15.

3. Sang T. Utility of low-copy nuclear gene sequences in plant phylogenetics. Crit Rev Biochem Mol Biol. 2002;37:121-47.

4. Álvarez I, Wendel JF. Ribosomal ITS sequences and plant phylogenetic inference. Plant Mol Evol. 2003:29:417-34.

5. Shaw J, Lickey EB, Beck JT, Farmer SB, Liu WS, Miller J, et al. The tortoise and the hare II: Relative utility of 21 noncoding chloroplast DNA sequences for phylogenetic analysis. Am J Bot. 2005;92:142-66.

6. Shaw J, Lickey EB, Schilling EE, Small RL. Comparison of whole chloroplast genome sequences to choose noncoding regions for phylogenetic studies in angiosperms: The tortoise and the hare III. Am J Bot. 2007;94:275-88.

7. Álvarez I, Costa A, Feliner G. Selecting single-copy nuclear genes for plant phylogenetics: A preliminary analysis for the Senecioneae (Asteraceae). J Mol Evol. 2008;66:276-91.

8. Clegg MT, Gaut BS, Learn GH, Morton BR. Rates and patterns of chloroplast DNA evolution. Proc Natl Acad Sci U S A. 1994;91:6795-801.

9. Naumann J, Symmank L, Samain M-S, Mueller KF, Neinhuis C, de Pamphilis $\mathrm{CW}$, et al. Chasing the hare - Evaluating the phylogenetic utility of a nuclear single-copy gene region at and below species level within the species rich group Peperomia (Piperaceae). BMC Evol Biol. 2011;11.

10. Zimmer EA, Wen J. Reprint of: Using nuclear gene data for plant phylogenetics: Progress and prospects. Mol Phylogenet Evol. 2013;66:539-50.

11. Song J, Shi L, Li D, Sun Y, Niu Y, Chen Z, et al. Extensive pyrosequencing reveals frequent intra-genomic variations of Internal Transcribed Spacer regions of nuclear ribosomal DNA. PLoS ONE. 2012;7:e43971.

12. Chase MW, Cowan RS, Hollingsworth PM, van den Berg C, Madriñán S, Petersen $\mathrm{G}$, et al. A proposal for a standardised protocol to barcode all land plants. Taxon. 2007;56:295-9.

13. Zhang N, Zeng L, Shan H, Ma H. Highly conserved low-copy nuclear genes as effective markers for phylogenetic analyses in angiosperms. New Phytol. 2012;195:923-37.

14. Duarte J, Wall PK, Edger P, Landherr L, Ma H, Pires JC, et al. Identification of shared single-copy nuclear genes in Arabidopsis, Populus, Vitis and Oryza and their phylogenetic utility across various taxonomic levels. BMC Evol Biol. 2010;10:61.

15. Harpke D, Meng S, Rutten T, Kerndorff H, Blattner FR. Phylogeny of Crocus (Iridaceae) based on one chloroplast and two nuclear loci: Ancient hybridization and chromosome number evolution. Mol Phylogenet Evol. 2013;66:617-27.

16. Lihova J, Shimizu KK, Marhold K. Allopolyploid origin of Cardamine asarifolia (Brassicaceae): Incongruence between plastid and nuclear ribosomal DNA sequences solved by a single-copy nuclear gene. Mol Phylogenet Evol. 2006;39:759-86
17. Meng J, Fougere-Danezan M, Zhang L-B, Li D-Z, Yi T-S. Untangling the hybrid origin of the Chinese tea roses: Evidence from DNA sequences of single-copy nuclear and chloroplast genes. Plant Syst Evol. 2011;297:157-70.

18. Tang L, Li J, Tan S, Li M-X, Ma X, Zhou Z-Q. New insights into the hybrid origin of Malus toringoides and its close relatives based on a single-copy nuclear gene Sbel and three chloroplast fragments. J Syst Evol. 2014;52:477-86.

19. Wang Z, Du S, Dayanandan S, Wang D, Zeng Y, Zhang J. Phylogeny reconstruction and hybrid analysis of Populus (Salicaceae) based on nucleotide sequences of multiple single-copy nuclear genes and plastid fragments. Plos One. 2014;9:e103645.

20. Cronn RC, Small RL, Haselkorn T, Wendel JF. Rapid diversification of the cotton genus (Gossypium: Malvaceae) revealed by analysis of sixteen nuclear and chloroplast genes. Am J Bot. 2002;89:707-25

21. Scherson RA, Choi HK, Cook DR, Sanderson MJ. Phylogenetics of New World Astragalus: Screening of novel nuclear loci for the reconstruction of phylogenies at low taxonomic levels. Brittonia. 2005;57:354-66.

22. Schlueter PM, Kohl G, Stuessy TF, Paulus HF. A screen of low-copy nuclear genes reveals the $L F Y$ gene as phylogenetically informative in closely related species of orchids (Ophrys). Taxon. 2007;56:493-504.

23. Fan $X$, Sha L-N, Yang R-W, Zhang H-Q, Kang H-Y, Ding C-B, et al. Phylogeny and evolutionary history of Leymus (Triticeae; Poaceae) based on a single-copy nuclear gene encoding plastid acetyl-CoA carboxylase. Bmc Evol Biol. 2009:9:247.

24. Naumann J, Salomo K, Der JP, Wafula EK, Bolin JF, Maass E, et al. Single-copy nuclear genes place haustorial Hydnoraceae within Piperales and reveal a Cretaceous origin of multiple parasitic Angiosperm lineages. Plos One. 2013:8:e79204.

25. Ness RW, Graham SW, Barrett SCH. Reconciling gene and genome duplication events: Using multiple nuclear gene families to infer the phylogeny of the aquatic plant family Pontederiaceae. Mol Biol Evol. 2011;28:3009-18.

26. Liu P-L, Wan Q, Guo Y-P, Yang J, Rao G-Y. Phylogeny of the genus Chrysanthemum L:: Evidence from single-copy nuclear gene and chloroplast DNA sequences. Plos One. 2012;7:e48970.

27. Liu Q, Liu H, Wen J, Peterson PM. Infrageneric phylogeny and temporal divergence of Sorghum (Andropogoneae, Poaceae) based on low-copy nuclear and plastid sequences. Plos One. 2014:9:e104933.

28. Naghavi MR, Rad MB, Riahi M, Taleie A. Phylogenetic analysis in some Hordeum species (Triticeae; Poaceae) based on two single-copy nuclear genes encoding acetyl-CoA carboxylase. Biochem Syst Ecol. 2013;47:148-55.

29. Rothfels CJ, Larsson A, Li F-W, Sigel EM, Huiet L, Burge DO, et al. Transcriptome-mining for single-copy nuclear markers in ferns. Plos One. 2013:8:UNSP e76957.

30. Salas-Leiva DE, Meerow AW, Calonje M, Griffith MP, Francisco-Ortega J, Nakamura K, et al. Phylogeny of the cycads based on multiple single-copy nuclear genes: Congruence of concatenated parsimony, likelihood and species tree inference methods. Ann Bot. 2013;112:1263-78.

31. Soltis DE, Gitzendanner MA, Stull G, Chester M, Chanderbali A, Chamala S, et al. The potential of genomics in plant systematics. Taxon. 2013;62:886-98.

32. Cacho NI, Burrell AM, Pepper AE, Strauss SY. Novel nuclear markers inform the systematics and the evolution of serpentine use in Streptanthus and allies (Thelypodieae, Brassicaceae). Mol Phylogenet Evol. 2014;72:71-81.

33. Lu Y, Ran J-H, Guo D-M, Yang Z-Y, Wang X-Q. Phylogeny and divergence times of Gymnosperms inferred from single-copy nuclear genes. Plos One. 2014;9:e107679.

34. Zeng L, Zhang Q, Sun R, Kong H, Zhang N, Ma H. Resolution of deep angiosperm phylogeny using conserved nuclear genes and estimates of early divergence times. Nat Commun. 2014:5:4956.

35. Sang T, Zhang D. Reconstructing hybrid speciation using sequences of low-copy nuclear genes: Hybrid origins of five Paeonia species based on Adh gene phylogenies. Syst Bot. 1999;24:148-63.

36. Egan AN, Schlueter J, Spooner DM. Applications of next-generation sequencing in plant biology. Am J Bot. 2012;99:175-85.

37. Lemmon EM, Lemmon AR. High-Throughput Genomic Data in Systematics and Phylogenetics. Annu Rev Ecol Evol Syst Vol 44. 2013:44:99-+.

38. Bräutigam A, Gowik U. What can next generation sequencing do for you? Next generation sequencing as a valuable tool in plant research. Plant Biol. 2010;12:831-41.

39. Tonnabel J, Olivieri I, Mignot A, Rebelo A, Justy F, Santoni S, et al. Developing nuclear DNA phylogenetic markers in the angiosperm genus Leucadendron (Proteaceae): A next-generation sequencing transcriptomic approach. Mol Phylogenet Evol. 2014;70:37-46. 
40. Pillon Y, Johansen J, Sakishima T, Chamala S, Barbazuk WB, Stacy EA. Primers for Low-Copy Nuclear Genes in the Hawaiian Endemic Clermontia (Campanulaceae) and Cross-Amplification in Lobelioideae. Appl Plant Sci. 2013;1:1200450.

41. Wickett NJ, Mirarab S, Nguyen N, Warnow T, Carpenter E, Matasci N, et al Phylotranscriptomic analysis of the origin and early diversification of land plants. Proc Natl Acad Sci. 2014;111:E4859-68.

42. Chamala S, García N, Godden GT, Krishnakumar V, Jordon-Thaden IE, Smet RD, et al. MarkerMiner 1.0: A New Application for Phylogenetic Marker Development Using Angiosperm Transcriptomes. Appl Plant Sci. 2015;3:1400115.

43. De Smet Y, Granados Mendoza C, Wanke S, Goetghebeur P, Samain M-S: Molecular phylogenetics and new (infra)generic classification to alleviate polyphyly in tribe Hydrangeeae (Hydrangeaceae, Cornales). Taxon accepted.

44. Samain M-S, Wanke S, Goetghebeur P. Unraveling extensive paraphyly in the genus Hydrangea s.l. with implications for the systematics of tribe Hydrangeeae. Syst Bot. 2010;35:593-600

45. Granados Mendoza C, Wanke S, Salomo K, Goetghebeur P, Samain M-S. Application of the phylogenetic informativeness method to chloroplast markers: A test case of closely related species in tribe Hydrangeeae (Hydrangeaceae). Mol Phylogenet Evol. 2013;66:233-42.

46. Samain M-S, Hernández Najarro F, Martínez Salas EM. First record of the critically endangered Hydrangea steyermarkii Standl. (Hydrangeaceae) in Mexico, and description of a new widespread Hydrangea species of Mesoamerica. Phytotaxa. 2014;162:181-97.

47. Morton CM. Newly sequenced nuclear gene (Xdh) for inferring Angiosperm phylogeny. Ann Mo Bot Gard. 2011;98:63-89.

48. Townsend JP. Profiling phylogenetic informativeness. Syst Biol. 2007:56:222-31.

49. Townsend JP, Su Z, Tekle YI. Phylogenetic signal and noise: Predicting the power of a dataset to resolve phylogeny. Syst Biol. 2012;61:835-49.

50. López-Giráldez F, Townsend J. PhyDesign: An online application for profiling phylogenetic informativeness. BMC Evol Biol. 2011;11:152

51. Ashburner M, Ball CA, Blake JA, Botstein D, Butler H, Cherry JM, et al. Gene ontology: Tool for the unification of biology. Nat Genet. 2000;25:25-9.

52. Jiao Y, Wickett NJ, Ayyampalayam S, Chanderbali AS, Landherr L, Ralph PE, et al. Ancestral polyploidy in seed plants and angiosperms. Nature. 2011;473:97-U113.

53. Han F, Peng Y, Xu L, Xiao P. Identification, characterization, and utilization of single copy genes in 29 angiosperm genomes. Bmc Genomics. 2014;15:504.

54. Renny-Byfield S, Wendel JF. Doubling down on genomes: Polyploidy and crop plants. Am J Bot. 2014;101(10):1711-25.

55. Amborella Genome Project. The Amborella genome and the evolution of flowering plants. Science. 2013:342:1467-+.

56. De Smet R, Adams KL, Vandepoele K, van Montagu MCE, Maere S, Van de Peer Y. Convergent gene loss following gene and genome duplications creates single-copy families in flowering plants. Proc Natl Acad Sci U S A. 2013;110:2898-903

57. Cerbah M, Mortreau E, Brown S, Siljak-Yakovlev S, Bertrand H, Lambert C. Genome size variation and species relationships in the genus Hydrangea. Theor Appl Genet. 2001;103:45-51.

58. Mortreau E, Siljak-Yakovlev S, Cerbah M, Brown SC, Bertrand H, Lambert C. Cytogenetic characterization of Hydrangea involucrata Sieb. and H. aspera D. Don complex (Hydrangeaceae): Genetic, evolutional, and taxonomic implications. Tree Genet Genomes. 2010;6:137-48.

59. Lysak MA. Live and let die: Centromere loss during evolution of plant chromosomes. New Phytol. 2014:203:1082-9.

60. Denda T: Cytological diversity of the genus Hydrangea (Saxifragaceae) in the Ryukyu Archipelago of Japan - On the origin of tetraploid Hydrangea liukiuensis endemic to Okinawajima Island. National University of Singapore and Tioman Island, Malasia: 21st COE Program of University of the Ryukyus; 2007:24

61. Reed SM, Jones KD, Rinehart TA. Production and characterization of intergeneric hybrids between Dichroa febrifuga and Hydrangea macrophylla. J Am Soc Hortic Sci. 2008;133:84-91.

62. Poptsova MS, Il'icheva IA, Nechipurenko DY, Panchenko LA, Khodikov MV, Oparina NY, et al. Non-random DNA fragmentation in next-generation sequencing. Sci Rep. 2014;4:4532.

63. Lemmon AR, Emme SA, Lemmon EM. Anchored hybrid enrichment for massively high-throughput phylogenomics. Syst Biol. 2012;61:727-44.

64. Rothfels CJ, Larsson A, Kuo L-Y, Korall P, Chiou W-L, Pryer KM. Overcoming deep roots, fast rates, and short internodes to resolve the ancient rapid radiation of eupolypod II ferns. Syst Biol. 2012;61:490-509.

65. Xiang JQ-Y, Thomas DT, Xiang QP. Resolving and dating the phylogeny of Cornales - Effects of taxon sampling, data partitions, and fossil calibrations. Mol Phylogenet Evol. 2011;59:123-38.
66. Hilu KW, Black CM, Oza D. Impact of gene molecular evolution on phylogenetic reconstruction: A case study in the Rosids (superorder Rosanae, Angiosperms). PLoS ONE. 2014;9:e99725.

67. Zwickl DJ, Hillis DM. Increased taxon sampling greatly reduces phylogenetic error. Syst Biol. 2002;51:588-98.

68. Xi Z, Liu L, Rest JS, Davis CC. Coalescent versus concatenation methods and the placement of Amborella as sister to water lilies. Syst Biol. 2014;63:919-32.

69. Doyle JJ, Doyle JL. A rapid DNA isolation procedure for small quantities of fresh leaf tissue. Phytochem Bull. 1987;19:11-5.

70. Stamatakis A. RAxML-VI-HPC: Maximum likelihood-based phylogenetic analyses with thousands of taxa and mixed models. Bioinformatics. 2006;22:2688-90.

71. Stamatakis A, Hoover $\mathrm{P}$, Rougemont J. A rapid bootstrap algorithm for the RAxML web servers. Syst Biol. 2008;57:758-71.

72. Rambaut A: FigTree Ver. 1.3.1. Http://tree.bio.ed.ac.uk/software/figtree/. 2009

73. Rambaut A, Charleston M: TreeEdit. 2002.

74. Sanderson M. A nonparametric approach to estimating divergence times in the absence of rate constancy. Mol Biol Evol. 1997;14:1218.

75. Pond SLK, Frost SDW, Muse SV. HyPhy: Hypothesis testing using phylogenies. Bioinformatics. 2005;21:676-9.

76. Bruno WJ, Socci ND, Halpern AL. Weighted neighbor joining: A likelihoodbased approach to distance-based phylogeny reconstruction. Mol Biol Evol. 2000;17:189-97.

77. Swofford DL. PAUP*. Phylogenetic Analysis Using Parsimony (*and Other Methods). Version 4. Sunderland, Massachusetts: Sinauer Associates; 2003.

\section{Submit your next manuscript to BioMed Central and take full advantage of:}

- Convenient online submission

- Thorough peer review

- No space constraints or color figure charges

- Immediate publication on acceptance

- Inclusion in PubMed, CAS, Scopus and Google Scholar

- Research which is freely available for redistribution 